\title{
Dissolution Highlights from the 2013 AAPS Annual Meeting in San Antonio
}

\author{
Nikoletta Fotaki ${ }^{1}$, Gregory P. Martin ${ }^{2, *}$, and Alger Salt ${ }^{3}$ \\ 'Department of Pharmacy and Pharmacology, University of Bath, Claverton Down, Bath, \\ $B A 27 A Y, U K$ \\ ${ }^{2}$ Complectors Consulting LLC, Pottstown, PA, USA \\ ${ }^{3}$ GlaxoSmithKline, Product Development, Research Triangle Park, NC, USA
}

e-mail:greg.martin@complectors.com

\begin{abstract}
A
\end{abstract} APS held its Annual Meeting and Exposition at the Henry B. Gonzalez Convention Center in San Antonio, Texas, during the week of November 10-14, 2013. The meeting is a premier gathering of pharmaceutical scientists from around the world and works to address the needs of the attendees, including members of over 40 focus groups in nine sections, or scientific disciplines, including two focused on dissolution testing: the In Vitro Release and Dissolution Testing and QbD and Product Performance groups. This year there were over 40 symposia, 16 roundtables, six short courses, nine open forums, and 15 sunrise sessions.

For those with an interest in dissolution testing, there was a pre-conference workshop on Developing a Biopharmaceutical Risk Assessment Road Map and symposia on Biorelevant In Vitro Performance Testing of Non-oral Dosage Forms, Emerging Dissolution Technologies, Understanding In vivo Dissolution: Can Imaging Tools Provide the Missing Link?, Must We Celebrate all Diversity? Regulatory Drivers and Perspectives on Differences in Requirements for Dissolution Release Methods and Specifications that Lead to Global Inconsistencies, and Challenges and Opportunities of Fast Dissolve Drug Delivery Systems. There were face-to-face meetings of the two focus groups mentioned and the Dissolution Discussion Group. See below for more details about some of these events.

\section{WORKSHOP ON DEVELOPING A BIOPHARMACEUTICS RISK ASSESSMENT ROADMAP}

This two-day, pre-conference workshop, organized by Maria Cruanes (Merck), Jennifer Dressman (University of Frankfurt), Annette Mullertz (University of Copenhagen), James Polli (University of Maryland), and Arzu Selen (FDA), was based on the thesis that a Biopharmaceutics Risk Assessment Road Map developed according to desired drug delivery patterns facilitates development and communication of linkages regarding the desired clinical outcomes. Arzu Selen opened the workshop by describing the goals, extensive breakout sessions, and deliverables, and discussed the four primary drug delivery based scenarios. Jack Cook (Pfizer) presented a case study for Rapid Therapeutic Onset using a drug for pain therapy, examining the effect of formulation variations on $T_{\max }$ and incorporating

${ }^{*}$ Corresponding author. observations that FDA will not give a claim of pain relief of less than $30 \mathrm{~min}$, effectively limiting the benefit from improving formulations to achieve actions shorter than the expected labeling. Tim Wigal (University of California, Irvine) discussed the need for Multi-Phasic Delivery in patients with attention deficit hyperactivity disorder (ADHD), where rapid onset with a decline, then subsequent increase in blood levels is needed. He showed how clinical data obtained in a laboratory school, where children were observed in a school-like setting, were used to optimize the formulation. The third case, Delayed/Targeted Therapeutic Onset, was presented by Jennifer Dressman. She discussed the concept of chronotherapeutics, citing examples where the incidence of heart attacks is highest around 9 a.m., while asthma attacks peak about 4 a.m., and gastric acid secretion peaks around 8 p.m., establishing the need for the delivery of certain types of drugs at certain times of day. Similarly, with diabetes it is important for the dose to be delivered at the right time to manage blood sugar levels. Alternatively, there are some diseases where the most important factor is the site of action, such as ulcers, Crohn's Disease, and colitis. In the case of colitis, the active drug should be released around the ileocecal valve to maximize delivery to the colon. Paul Dickinson (Astra Zeneca) presented the fourth case, Exposure-Based Targeted Steady-State or Trough $\left(C_{\min }\right)$ Plasma Concentrations. He cited drugs such as antipsychotics, antibiotics, and oncology drugs as falling into this category. In these cases, pharmacokinetic data are often used to decide when a formulation is sufficiently developed.

Day two of the workshop consisted of breakout sessions where presenters and participants discussed various drug delivery patterns. Discussed in each breakout session were what information is needed for success, what models were most appropriate (animal, in vitro, in silico, etc.), what technologies or formulation strategies work best, and what are the most critical clinical or preclinical tests. The organizers plan to present the findings at a subsequent workshop.

\section{CHALLENGES AND OPPORTUNITIES OF FAST-DISSOLVE DRUG DELIVERY SYSTEMS}

Suniket Fulzele (CIMA Labs) moderated this minisymposium on fast-dissolve systems. Suniket pointed out the advantages such as ease of swallowing and improved 
compliance, the challenges such as stability and tastemasking, and the opportunities such as product lifecycle extension, pediatric products, and product differentiation. Rick Chan (Lohmann Therapeutic Systems, Inc.), in a presentation titled "Formulation Challenges and Approaches for Orally Disintegrating Tablets Oral Thin Films: New Avenues and Strategies," discussed the variety of products now available as thin films and the advantages of transmucosal or buccal absorption, especially for avoiding hepatic first-pass effects. Some of the challenges for these products are the limited ability to affect bioavailability and bioequivalence and to increase drug loading. Trends and Opportunities in Pediatric Development were discussed by Daniel Schaufelberger (Janssen). He described the need to identify what drugs work in children and then find the right dose and an age-appropriate formulation, remembering that they are not just small adults. These might include smaller tablets, orally disintegrating tablets, thin films, or liquids. The strategy may be affected by whether it is a line extension of a drug developed for adults or one where pediatrics is the primary population, and whether exclusivity is possible or it is a generic or OTC product. Within the younger population, there are subpopulations of neonates, infants, children, and adolescents that may have different needs. In this area, there is also an opportunity for synergy with products for the geriatric population.

\section{BIORELEVANT IN VITRO PERFORMANCE TESTING OF NON-ORAL DOSAGE FORMS}

This symposium was moderated by Poonam Delvadia (Virginia Commonwealth University) and Stephen Mayock (Collegium Pharmaceutical). Thomas Karnes (Virginia Commonwealth University) talked about "Biorelevant Dissolution Approaches for Subcutaneous and Buccal Application Sites." After a general introduction to biorelevant dissolution testing, he presented a case of biorelevant, accelerated dissolution of a naltrexone implant with the use of the Cellmax Capillary System (a miniaturized bioreactor used for cell culture) that mimics the subcutaneous vasculature around the implant and the flow rate. This methodology led to the development of an in vitro-in vivo relationship that was compared with the modified flow-through methodology that is currently used for these cases. In the second part of his presentation, he discussed biorelevant dissolution methods for oral transmucosal formulations and a case study of nicotine dissolution from a smokeless tobacco product (Snus) with the use of a bidirectional transmucosal apparatus. Biorelevant in vitro release test methods for semisolid dosage forms were discussed in the following presentation by Kailas Thakker (Tergus Pharma). The factors affecting the release of an API from a semisolid dosage form and the development of a release test using vertical diffusion cells were described. She then touched on the QbD approach for the development of a release test for semisolid formulations by presenting some examples. Her presentation concluded by settling the question on the appropriate criteria to be used for equivalence when using in vitro release testing. Anne Seidlitz (University of Greifswald) gave a presentation on biorelevant in vitro performance testing of drug-eluting stents (DES). After describing the aspects of coronary stents, she emphasized the need for a predictive in vitro test due to the drawbacks of the relevance of in vivo plasma concentration data and animal models. The general issues on the methodology used for DES release testing, including the use of surrogates or intermediate products, stent expansion, sink conditions, adsorption and instability, and accelerated testing conditions were noted, and a detailed methodology regarding the choice of media and apparatus on the development of a release test was given. She highlighted the fact that release studies with the sample and separate methods (small volume incubation setups, reciprocating holder [USP 7], flowthrough cell) are conducted in stirred media, whereas in vivo DES are in direct contact with vessel wall tissue. The use of hydrogels as a first approach to simulate tissue was proposed, and release studies with the flow-through cell (USP 4) accommodating a gel compartment were presented. Finally, she talked about further developments of this approach in terms of gel modifications and alternate gelling agents. The last presentation in this symposium, given by Tapash Ghosh (FDA), was on the FDA perspective on the topic. He first described the expectations of biorelevant release testing and then noted that the development of in vitro release testing for non-oral dosage forms may follow the general principles of dissolution tests for solid oral dosage forms, but the different characteristics of the novel/special dosage forms and the different sites and modes of administration should be considered. He gave some hints for the development of in vitro release testing of several non-oral dosage forms and the expectations from a regulatory point of view. He pointed out that in vitro release methods should be relatively easy to perform and ideally should be predictive of in vivo release, and when an IVIVC can be developed, it is suggested that this be discussed with FDA beforehand.

\section{MUST WE CELEBRATE ALL DIVERSITY? REGULATORY DRIVERS AND PERSPECTIVES ON DIFFERENCES IN REQUIREMENTS FOR DISSOLUTION RELEASE METHODS AND SPECIFICATIONS THAT LEAD TO GLOBAL INCONSISTENCIES}

Paul Dickinson (Astra Zeneca) and Jack Cook (Pfizer), as moderators, set the stage for this roundtable by noting the general aspects and challenges of dissolution in terms of ensuring bioequivalence among batches, monitoring the manufacturing process, and the traditional "quality aspects." It was emphasized that interpretation of the relevant, sometimes complicated, data set may depend on which of the above aspects is most important to whoever is looking at the data. Angelica Dorantes (FDA) discussed 
dissolution method development for meaningful in vitro dissolution/release tests and performance specifications and the timing of development with the aspect of successful QbD implementation. She described the three approaches used for clinically relevant dissolution and specifications: based on in vitro consideration only, in vivo BE data available, in vitro-in vivo correlation model available. Finally, she highlighted the use of dissolution under $\mathrm{QbD}$ paradigm. The EU regulatory requirements on dissolution were presented by Evangelos Kotzagiorgis (EMA). He presented briefly the dissolution method development and the acceptance criteria as described in the European Pharmacopoeia, and he commented on their clinical relevance. By pointing out the relevant guidelines in which dissolution testing is referenced, he raised the question "Does one method fit all?" The philosophy of dissolution testing in Japan was presented by Shunji Nagata (Hiroshima International University). He emphasized the importance of dissolution testing and the efforts made for international harmonization, although differences still exist. The major differences in the Japanese Pharmacopoeia (JP) relate to the compendial apparatus, the dissolution media, and the procedure. For example, the reciprocating cylinder and the simulated fluids are not included in JP16. In particular, he discussed the fact that the addition of enzymes, such as pepsin or pancreatin, to the dissolution medium is not recommended based on bioinequivalence obtained between raw gelatin capsules and cross-linked gelatin capsules. An interesting discussion of the challenges faced when proposing and agreeing on a release method and specification across the globe followed the presentations.

\section{EMERGING DISSOLUTION TECHNOLOGIES}

Alger Salt (GSK) and Nikoletta Fotaki (University of Bath) moderated this mini-symposium on Emerging Dissolution Technologies. Xujin Liu (BMS) discussed the use of lowfield nuclear magnetic resonance (NMR), Fourier transform infrared spectroscopy (FTIR), and ultraviolet (UV) imaging of the dissolution process in a presentation on in vitro dissolution imaging. Imaging technologies can provide "another set of eyes" to better enable dissolution scientists to understand and characterize the release mechanisms of drugs from dosage forms. These technologies are typically used in systems that employ flow-through, as opposed to stirred, media. Raman spectroscopy could also be employed for characterizing drug precipitation processes from both dosage forms and different forms of active pharmaceutical ingredients. NMR is used to monitor the ingress of water into a dosage form. UV imaging is generally used to monitor the movement and concentration of a dissolved drug. Matt Burke (GSK) spoke about a device that can be installed on a conventional dissolution ap- paratus that allows it to mimic the peristaltic action within the gastrointestinal tract. Case studies were presented that showed superior in vitro-in vivo correlation results when compared with work done using the same apparatus without the peristaltic device. This was shown for both modified-release and at least one immediate-release formulation. The device can be adjusted to operate in ways that make it more relevant to the more dominant peristalsis observed in the fed state or likewise in the fasted state. The device will soon be commercially available. Anette Mullertz (University of Copenhagen) presented results of work done using the Dynamic Gastric Model, which provides a much more diverse set of conditions for the dosage form to be exposed to within the stomach. Acid secretion from the stomach wall and the slow rate of mixing cause a significant variation of $\mathrm{pH}$ in the fed stomach, which is difficult to simulate with conventional dissolution test conditions and equipment. The stomach enzyme lipase can affect dissolution of products that contain lipids, but this is usually omitted from simulated gastric fluids. The Dynamic Gastric Model provides these and other conditions not available with conventional dissolution test equipment. Superior correlations were demonstrated in case studies versus those obtained with conventional in vitro dissolution tests. The device itself is not commercially available, but a service is provided whereby samples can be processed with the Dynamic Gastric Model on a contract basis.

\section{IVRDT FOCUS GROUP FACE-TO-FACE MEETING}

The annual face-to-face meeting of the focus group was held at midday on Monday, November 11. Attendance by over 70 people probably represented the largest turnout for an annual meeting of this group and was likely facilitated by a new aspect: a presentation by the focus group's student representative, Trinh Vo, who is pursuing her Ph.D. at Mercer University. Incoming chair Xujin Lu (BMS) and outgoing chair Greg Martin (Complectors Consulting) reviewed the accomplishments of the group, which included development of programming for the AAPS Annual Meeting and Exposition, the National Biotechnology Conference, and the Eastern Analytical Symposium; updating of the website to provide significantly more content for those interested in dissolution; continuation of the global outreach efforts with a two-day workshop on Dissolution and Bioequivalence at la Plate, Argentina; and presentation of a webinar on Approaches to the Investigation of Dissolution Changes and Failures conducted by Jianmei Kochling (Genzyme). Trinh Vo (Mercer University) presented the results from a survey of graduate students from several universities, detailing their interests and providing valuable input, which fueled the website update. 